\title{
Operationalizing Diversity: Population and Patient-Specific Competencies for Health Service Psychologists
}

\author{
Morgan T. Sammons
}

Published online: 19 October 2021

(c) National Register of Health Service Psychologists 2021

Awareness of the need for multicultural training has become an essential hallmark of health provider education over the past four decades. From early initiatives springing from the civil rights and women's rights movements of the 1960s to the establishment of a more systematic multicultural pedagogy in the 1980s, diversity education is now firmly incorporated into the curriculum. A commitment to diversity is a requirement for program accreditation by the American Psychological Association's Commission on Accreditation (American Psychological Association, 2021). It is also a standard for medical education in the US (American Association of Medical Colleges, 2021). This commitment is mirrored in accreditation standards for other health and mental healthcare providers. Diversity, however, is not a unitary construct, and as our understanding of the needs of culturally diverse individuals deepens, its definition will attract ongoing controversy. A shift in focus from population-based definitions of diversity to our ethical obligation to thoroughly assess individual patient needs somewhat removes us from the larger debate. At the same time, it heightens our responsibility to define the competencies necessary to treat individuals from diverse backgrounds. In other words, the challenge has become not how we handle population-specific diversity but how we can adequately train practitioners to wholly recognize the diverse characteristics of the patient sitting in front of us.

Few areas of practice have more significant consequences than the assessment of cognitive decline, dementia, or Alzheimer's disease. While bilingual capabilities have been considered a protective factor in certain dementias, such capacities may affect performance on non-verbal and verbal tests. Bilingualism may also obscure certain features of Alzheimer's disease, making the accurate assessment of bilingual capabilities an essential component of diagnosis. If the hypothesis put forth by Díaz-Santos et al. (2021) is correct, linguistic competencies (e.g., monolingualism or bilingualism) affect the clinical presentation of Alzheimer's disease. Thus, the clinician is faced with decisions involving the choice of assessment tools and interpreting those results. This clinical decision-making is especially pertinent given the results of another review that found differences in sensitivity and specificity for "gold standard" neuropsychological assessment devices, both verbal and non-verbal, between Spanish and English speakers (Burke et al., 2019). Díaz-Santos et al. report that a high percentage of Spanishspeaking patients referred for culturally competent services have previously been evaluated only in English, raising the question of whether the performance of such evaluations is in keeping with ethical standards of practice.

No discussion of cultural competence, however, can take place without an acknowledgment of the mediating effects of ethnicity and related individual characteristics on the utilization of telepsychology, an increasingly dominant mode of psychological service delivery. Several recent analyses of telehealth usage have revealed that Asian, Black/African American, and Hispanic/Latino ethnic identification has been associated with negative rates of telehealth utilization. This difference holds even when considering other non-ethnically mediated factors such as increased age, rural status, and lower income (Eberly et al., 2020; Hsiao et al., 2021).

There is a dearth of information regarding telehealth utilization among transgender and gender-diverse populations. As Pankey et al. (2021) observed, little attention has been paid to this population during the pandemic. Nonetheless, there are aspects of telehealth that may make it an attractive option for such patients. Unsurprisingly, experiences of discrimination have been demonstrated to reduce transgender individual's utilization of in-person emergency care in at least one small study (Samuels et al., 2018). Sequiera et al. (2021) reported that approximately half of transgender youth already receiving services in a gender clinic were amenable to telepsychological interventions. Still, no information is 
available regarding telehealth preferences for transgender and gender-diverse individuals who have not yet engaged in care. Because competent care for transgender and genderdiverse populations remains scarce in the healthcare community at large, telepsychology, if provided by those with the unique skill set required to treat this population, may be an effective mechanism of expanding access to care.

As I've noted, codifications of diversity that began with the women's and civil rights movements have faced some opposition. Those opposed to such codification frequently argued that these distinctions less afforded protections than they extended "special rights" to certain classes of people. While such opposition has often targeted sexual minorities, it may be that the emerging label of "twice exceptional" children (Lewis, 2021) will attract similar negative attention, inasmuch as it can make a new group of children eligible for potentially expensive and scarce special education services. Lewis reminds us that the definition itself is not without controversy, but this may be expected for any emerging conceptualization that has received little academic scrutiny in the past. Even so, there exists a cohort of schoolchildren who possess both exceptional abilities and manifest signs of a defined learning disability. As our understanding of diversity becomes more nuanced, it behooves us to understand that exceptional ability and disability are not unipolar but indeed are likely to co-exist in the same student. This understanding is a rather common-sense observation that we have little trouble applying to adults. Still, its rigorous assessment in children will require a more nuanced approach than that of standard measures of intelligence, attention, or academic ability. The correct selection of assessment instruments that can capture the spectrum of ability and disability must be carefully chosen, as Lewis underscores, with special attention to the requirements of those who have been historically disadvantaged by standardized assessments.

Self-harm behavior in adolescents often accompanies emotional dysregulation and a diagnosis of borderline personality disorder (Boylan \& Conley, 2021), but it is essential to understand that the pathogenesis of such behavior is variable. Self-harm behavior is understudied in male populations and, in some male samples, is modulated by both life stressors and, as might be expected, age and other individual characteristics (Steinhoff et al., 2020). More recently, linkages have been observed between COVID-19, either via neurological and psychological effects of infection or societal stressors associated with pandemic restrictions (Banerjee et al., 2021). Thus, presumptions about individual psychopathology must be tempered with the knowledge of the effects of group belongingness, potential neuropsychological compromise associated with infection, and societal stressors as possible contributors to self-harm behavior.

As always in our fall issue, Dean Steve Smith (2021) presents an analysis of the decisions with mental health implications by the Supreme Court in its most recent term. Although Dean Smith notes it was a term of highly charged political issues, opening with the confirmation hearings of Justice Barrett and the specter that the Court would be called upon to decide the presidential election, this term had somewhat fewer cases with direct implications for mental health law. Among these, in Jones v. Mississippi, was a clarification of the conditions under which a judge might sentence a juvenile to a life sentence without the possibility of parole. The majority ruled that a determination of "permanent incorrigibility" need not be made prior to imposing such a sentence. To many, this decision reflects a distinction without a difference, in that the United States is alone among all nations in permitting those under 18 to be sentenced to life without the possibility of parole. Whether one maintains moral opposition to this stance or not, Dean Smith reminds us that mental health practitioners are often called on in such cases, and the quality of their examination of juveniles facing such peril is paramount. Dean Smith also noted that while the Court did not accept a case involving solitary confinement, the Court received several amicus curiae briefs arguing that this practice represents cruel and unusual punishment. While there is an encouraging trend for more US jurisdictions to move away from solitary confinement, it remains disproportionally used among certain populations, including those with mental illness. Perhaps in a future term, the Court will see fit to address this abhorrent practice.

\section{References}

American Association of Medical Colleges, Liaison Committee on Medical Education (2021). Functions and Structure of a Medical School. AAMC: Washington, DC.

American Psychological Association, Commission on Accreditation (2021). Standards of accreditation for health service psychology and accreditation operating procedures. APA: Washington, DC.

Banerjee, D., Kosagisharif, J. R., \& Rao, S. (2021). 'The dual pandemic' of suicide and COVID-19: A biopsychosocial narrative of risks and prevention. Psychiatry Research, Jan;295:113577. https://doi.org/10.1016/j.psychres.2020.113577. Epub 2020 Nov 18.

Boylan, K. \& Conley, C. (2021). A Framework for Self-Harm Cessation in Adolescents. Journal of Health Service Psychology, 47(4). https://doi.org/10.1007/s42843-021-00051-4.

Burke, S. L., Naseh, M., Rodriguez, M. J., Burgess, A., \& Loewenstein, D. (2019). Dementia-related neuropsychological testing considerations in non-Hispanic white and Latino/Hispanic populations. Psychology and Neuroscience, 12, 144-168.

Díaz-Santos, M., Yañez, J., \& Suarez, P. A. (2021). Alzheimer's Disease in Bilingual Latinos: Clinical Decisions for Diagnosis and Treatment Planning. Journal of Health Service Psychology, 47(4). https://doi.org/10.1007/s42843-021-00050-5.

Eberly, L. A., Kallan, M. J., Julien, H. M., Haynes, N., Khatana, S. A. M., et al (2020). Patient Characteristics Associated With Telemedicine Access for Primary and Specialty Ambulatory Care 
During the COVID-19 Pandemic. JAMA Network Open, Dec 1: 3(12), e2031640.

Hsiao, V., Chandering, T., Lankton, R. L., Huebner, J. A., Baltus, J. J., et al (2021). Disparities in Telemedicine Access: A CrossSectional Study of a Newly Established Infrastructure during the COVID-19 Pandemic. Applied Clinical Informatics, 12, 445-458, https://doi.org/10.1055/s-0041-1730026. Epub 2021 Jun.

Lewis, L. R. (2021). Twice-Exceptionality: Maximizing Academic \& Psychosocial Success in Youth. Journal of Health Service Psychology, 47(4). https://doi.org/10.1007/s42843-021-00046-1

Pankey, T. L., Heredia, D., Vencill, J. A., Gonzalez, C. A. (2021). Gender-Affirming Telepsychology During and After the COVID-19 Pandemic: Recommendations for Adult Transgender and Gender Diverse Populations. Journal of Health Service Psychology, 47(4). https://doi.org/10.1007/s42843-021-00048-z

Samuels, E. A., Tape, C., Garber, N., Bowman, S., Choo, E. K. (2018). Sometimes you feel like a freak show: A qualitative assessment of emergency care experiences among transgender and gender-nonconforming patients. Annals of Emergency Medicine, 71, 170-182.
Sequiera, G. M., Kidd, K. C., Coulter, R. W. S., Miller, E., Fortenberry, D., Garofalo, R., et al. (2021). Transgender youth's perspective on telehealth for delivery of gender-affirming care. Journal of Adolescent Health, 68, 1207-1210.

Smith. S. R. (2021). A Pandemic Term With "Highly Charged Issues": The U.S. Supreme Court 2020-2021. Journal of Health Service Psychology, 47(4). https://doi.org/10.1007/s42843-021-00047-0

Steinhoff, A.,Bechtiger L., Ribeaud, D., Eisner, M., \& Shanahan, L. (2020). Stressful life events in different social contexts are associated with self-injury from early adolescence to early adulthood. Frontiers in Psychiatry, 11, 478200, https://doi.org/10.3389/fpsyt. 2020.487200

Morgan T. Sammons, $\mathrm{PhD}$, ABPP, is the $\mathrm{CEO}$ of the National Register of Health Service Psychologists, and the Editor in Chief of the Journal of Health Service Psychology. He is a retired Navy captain and was formerly the U.S. Navy's specialty leader for clinical psychology. 